USM-TH-106

\title{
Photo-production of Nucleon Resonances and Nucleon Spin Structure Function in the Resonance Region
}

\author{
Di Qing ${ }^{1,2}$ and Iván Schmidt ${ }^{1}$ \\ ${ }^{1}$ Departamento de Física, Universidad Técnica Federico Santa María, Casilla 110-V, Valparaíso, \\ Chile \\ ${ }^{2}$ Institute of Modern Physics, Southwest Jiaotong University, Chengdu, 610031, China
}

\begin{abstract}
The photo-production of nucleon resonances is calculated based on a chiral constituent quark model including both relativistic corrections $H_{\text {rel }}$ and twobody exchange currents, and it is shown that these effects play an important role. We also calculate the first moment of the nucleon spin structure function $g_{1}\left(x, Q^{2}\right)$ in the resonance region, and obtain a sign-changing point around $Q^{2} \sim 0.27 \mathrm{GeV}^{2}$ for the proton.
\end{abstract}

Typeset using REVTEX 


\section{INTRODUCTION}

A major goal of hadron physics is to understand the spectrum, structure, and interactions of hadrons as a consequence of the strong interactions. However, quantum chromodynamics (QCD), the fundamental theory for strong interactions, is so difficult to solve in the nonperturbative region of low-energy hadron physics that no one is currently able to quantitatively describe the hadron characteristics from first principles. Because of this, effective models are built, trying to keep the essential features of the underlying basic theory, and which hopefully can be solved or at least approximated in a satisfactory way. Among these models, the constituent quark model is the most successful. Nevertheless, it is important to extend its range of application beyond the traditional static hadronic properties, such as the mass spectrum, to processes that can provide information in a more stringent way about the detailed quark dynamics. Electromagnetic transitions play in this respect a special role,

since the photon is a particularly clean probe. On the experimental side, the opening of CEBAF will certainly bring more precise experimental data in the near future.

In its application to the photo-production of nucleon resonances, the non-relativistic quark model had to be modified by adding some terms in order to have a consistent theory. In fact, the study of the photo-production of nucleon resonances has a long history [1]. In the non-relativistic quark model, it is begun with the work of Koniuk and Isgur, based on one-body reduction current [2]. Later on, Close and Li stressed the importance of spin-orbit and non-additive terms associated with the Wigner rotation of the quark spin transformed from the frame of the recoiling quark to the frame of the recoiling baryon, and also the role of the binding potential [3] and configuration mixing [4]. Since the spin-orbit (S-L) term of one-gluon exchange potential [5] was ignored in the investigation of baryon spectrum in the Isgur model [6], most of the authors did not consider the two-body exchange currents corresponding to the one-gluon exchange potential in the calculation of resonance photon decay. However, the S-L term is important in the study of baryon-baryon interactions. Some authors also included it in order to investigate the baryon spectroscopy [7]. 
On the other hand, chiral symmetry is probably the most important feature of QCD in the nonperturbative region [8]. This is introduced in the quark model by adding one-pion and one-sigma exchange potentials between constituent quarks. Recently, based on this chiral constituent quark model, Buchmann et al. investigated the electromagnetic properties of octet and decuplet baryons, including the two-body gluon and meson exchange currents. In their calculations, the two-body exchange currents play an important role in the explanation of the nonzero $\gamma N \rightarrow \Delta E 2$ transition [9] and the neutron charge radius [10], and also in order to fit the electromagnetic properties of the octet and decuplet baryons [11]. Meyer et al. included the two-body exchange currents to study the photo-production of the resonance states below $\sqrt{s}=1.6 \mathrm{GeV}$ and obtained a reasonable good agreement with experiment [12].

In the first part of this work, based on the chiral constituent quark model [10], we go beyond the work of Meyer et al. [12], calculating the photo-production amplitudes of nucleon resonances up to energies higher than they do, that is up to $2 \mathrm{GeV}$, and we also include the contributions of the spin-orbit and non-additive terms, in addition to the contributions of the two-body exchange currents.

The effect of nucleon resonances is also important in other electromagnetic transition processes. In fact, recently there has been much interest in the nucleon spin structure function $g_{1}\left(x, Q^{2}\right)$ in the low energy region, where it is mainly saturated by the low-lying resonances. For the proton, since $g_{1}\left(x, Q^{2}\right)$ satisfies a positive sum rule in the large $Q^{2}$ limit, and the negative Gerasimov-Drell-Hearn (GDH) sum rule in the real photon limit [13], there should be a sign-changing in the low $Q^{2}$ region. Theoretically, phenomenological models 14 17] have been proposed to study the $Q^{2}$ evolution of the first moments of the nucleon spin structure function in the resonance region. After taking into account the nucleon resonances explicitly, the predictions [14 are in good agreement with the few data points of the SLAC experiment E143, although within large experimental errors [18], and gave a sign-changing point at a smaller $Q^{2} \sim 0.22 \mathrm{GeV}^{2}$, instead of $0.8 \mathrm{GeV}^{2}$ 19 that was predicted before. Li and Dong considered the interference cross section between transverse and longitudinal currents based on a non-relativistic constituent quark model. In their calculation, the contribution of 
the interference cross section pushes the sign-changing point to around $0.53 \mathrm{GeV}^{2}$ 15. After including the background contribution, the sign-changing point appears at a smaller energy

scale, about $0.3 \mathrm{GeV}^{2}$ [16]. Soffer and Teryaev introduced a parametrization to investigate the $Q^{2}$ dependence of GDH sum rule [20]. All these calculations indicate that there is a very strong $Q^{2}$ dependence behavior on the evolution of the nucleon spin structure function due to the resonance contributions. In order to reliably perform the saturation, it is necessary to take into account all kinds of effects carefully, and that is what we plan to do here.

In section II of this paper, we calculate the photo-production amplitudes of nucleon resonances up to energies of $2 \mathrm{GeV}$, including both the relativistic corrections and the twobody exchange currents. Furthermore, we also study the effects of the two-body exchange currents in the nucleon spin structure function and GDH sum rule in the resonance region in section III. Finally a summary and conclusions are given in section IV.

\section{THE PHOTO-PRODUCTION OF NUCLEON RESONANCES}

The helicity amplitudes of nucleon excitations are defined as

$$
A_{\lambda}=\left\langle R, J^{\prime}, \lambda\left|H_{T}\right| N, \frac{1}{2}, \lambda-1\right\rangle
$$

where $\lambda$ is the helicity of resonance $R$ with total angular moment $J^{\prime}$. The electromagnetic transition operator $H_{T}$ is composed of one-body non-relativistic reduction term, spin-orbit and non-additive terms (the last two terms, first introduced by Brodsky and Primack [21], are called collectively as $H_{\text {rel }}$ ) [3],

$$
H_{T}=H_{N R}+H_{S O}+H_{N A}
$$

In fact, it is more convenient to calculate the transition amplitudes in the form of current matrix elements. For real photons, we have

$$
A_{\lambda}=-\sqrt{\frac{2 \pi}{\omega}}\left\langle R, J^{\prime}, \lambda|\vec{\epsilon} \cdot \vec{j}(\vec{q})| N, \frac{1}{2}, \lambda-1\right\rangle,
$$


in which $\vec{\epsilon}=-\frac{1}{\sqrt{2}}(1, i, 0)$ is the photon transverse polarization vector, $(\omega, \vec{q})$ is the four momentum transfer of the photon in the center of mass frame, $\vec{j}(\vec{q})$ is the electromagnetic current corresponding to the transition operator $H_{T}$, and which can be easily obtained from $H_{T}$ as:

$$
\begin{aligned}
\vec{j}(\vec{q}) & =\vec{j}_{N R}(\vec{q})+\vec{j}_{O S}(\vec{q})+\vec{j}_{N A}(\vec{q}), \\
\vec{j}_{N R}(\vec{q}) & =\sum_{i=1}^{3} \mu_{i}\left(i\left[\vec{\sigma}_{i} \times \vec{p}_{i}, e^{i \vec{q} \cdot \vec{r}_{i}}\right]+\left\{\vec{p}_{i}, e^{i \vec{q} \cdot \vec{r}_{i}}\right\}\right), \\
\vec{j}_{S O}(\vec{q}) & =\sum_{i=1}^{3}-\frac{1}{2}\left[2 \mu_{i}-\frac{e_{i}}{2 m}\right] \frac{\omega}{2 m_{i}} i\left\{\vec{\sigma}_{i} \times \vec{p}_{i}, e^{i \vec{q} \cdot \vec{r}_{i}}\right\}, \\
\vec{j}_{N A}(\vec{q}) & =\sum_{i<j}^{3} \frac{\omega}{4 M_{T}} i\left(\frac{\vec{\sigma}_{i}}{m}-\frac{\vec{\sigma}_{j}}{m}\right) \times\left(e_{j} e^{i \vec{q} \cdot \vec{r}_{j}} \vec{p}_{i}-e_{i} e^{i \vec{q} \cdot \vec{r}_{i}} \vec{p}_{j}\right) .
\end{aligned}
$$

Here, $e_{i}, \vec{p}_{i}, \vec{r}_{i}, \mu_{i}$ are the charge, momentum, position and magnetic moment of the $i$ th quark, $m$ and $M_{T}$ are the quark and nucleon mass, and $A_{i}, \phi_{i}, B_{i}$ and $E_{i}$ are the electromagnetic fields. According to Eq.(可), we can see that $\vec{j}_{N R}$ is exactly the lowest order non-relativistic one-body electromagnetic current reduction. With the multipole expansion of electromagnetic current 22

$$
j(\vec{q} ; m)= \begin{cases}\frac{\omega}{|\vec{q}|} \sqrt{4 \pi} \sum_{J \geq 0} i^{J} \sqrt{2 J+1} \hat{M}_{J m}(|\vec{q}|) & m=0 \\ -\sqrt{2 \pi} \sum_{J \geq 1} i^{J} \sqrt{2 J+1}\left(\hat{T}_{J m}^{\mathrm{el}}(|\vec{q}|)+m \hat{T}_{J m}^{\mathrm{mag}}(|\vec{q}|)\right) & m= \pm 1\end{cases}
$$

in which $\hat{M}_{J}, \hat{T}_{J}^{\mathrm{el}}$ and $\hat{T}_{J}^{\mathrm{mag}}$ are Coulomb, electric and magnetic multipole operators of rank $J$, respectively, we have

$$
\begin{aligned}
A_{\lambda}= & \frac{2 \pi}{\sqrt{\omega}} \sum_{J \geq 1} i^{J} \sqrt{2 J+1}\left(\left\langle R, J^{\prime}, \lambda\left|\hat{T}_{J 1}^{\mathrm{el}}(|\vec{q}|)\right| N, \frac{1}{2}, \lambda-1\right\rangle\right. \\
& \left.+\left\langle R, J^{\prime}, \lambda\left|\hat{T}_{J 1}^{\mathrm{mag}}(|\vec{q}|)\right| N, \frac{1}{2}, \lambda-1\right\rangle\right) .
\end{aligned}
$$

Due to the parity and angular momentum conservation, only one electric and one magnetic multipole operators contribute to the helicity amplitudes.

In this work we study the photo-production amplitudes of nucleon resonances based on the chiral constituent quark model [10], which includes not only the one-gluon exchange potential, but also the one-pion and one-sigma exchange potentials to model chiral symmetry 
breaking of QCD in the nonperturbative region. As pointed out in the introduction, the S-L term in the one-gluon exchange potential is important in baryon-baryon interactions. Furthermore, when it is included in the study of the baryon spectrum, and because of gauge invariance, it is necessary to consider its effects, namely the two-body gluon exchange current $\vec{j}_{g q \bar{q}}\left(\vec{r}_{i}, \vec{r}_{j}, \vec{q}\right)$, which turns out to be important in the investigation of the electromagnetic transitions 10]. There exist several methods to derive the two-body gluon exchange current, such as the minimal electromagnetic coupling method and the Feynman diagram method. Buchmann et al. derived it by the Feynman diagram approach [10. In addition, the gauge invariant currents corresponding to the one-pion exchange potential are the pion-pair current $\vec{j}_{\pi q \bar{q}}\left(\vec{r}_{i}, \vec{r}_{j}, \vec{q}\right)$ and the pionic current $\vec{j}_{\gamma \pi \pi}\left(\vec{r}_{i}, \vec{r}_{j}, \vec{q}\right)$, which are also derived using Feynman diagrams. Explicit expressions for all these two-body exchange currents can be found in Ref. [10].

In Ref. [3], the quark mass was treated as free parameter in order to include the contributions of the scalar potentials. Here, we will investigate the contributions of the scalar potentials carefully. Buchmann et al. found that the scalar potentials contribute significantly to the electromagnetic properties of the octet and decuplet baryons. According to Eq.(2.10) of Ref. [3], to $O\left(1 / m^{2}\right)$ we have

$$
\vec{j}_{\text {scalar }}\left(\vec{r}_{i}, \vec{r}_{j}, \vec{q}\right)=-\frac{i}{2 m^{2}}\left\{e_{i} e^{i \vec{q} \cdot \vec{r}_{i}} \vec{\sigma}_{i} \times \vec{q} V^{\text {scalar }}\left(\vec{r}_{i}, \vec{r}_{j}\right)+(i \leftrightarrow j)\right\} .
$$

In the chiral constituent quark model [10], the scalar potentials include a two-body harmonic oscillator confinement potential $V^{\text {conf }}\left(\vec{r}_{i}, \vec{r}_{j}\right)$ and a one-sigma exchange potential $V^{\sigma}\left(\vec{r}_{i}, \vec{r}_{j}\right)$.

Thus, in the chiral constituent quark model [10], the total electromagnetic current $\vec{j}_{\text {tot }}$, which satisfies the gauge invariance condition, should be given by a sum of one-body nonrelativistic reduction, spin-orbit, non-additive, and two-body exchange currents:

$$
\begin{aligned}
\vec{j}_{\text {tot }}= & \vec{j}_{N R}(\vec{q})+\vec{j}_{O S}(\vec{q})+\vec{j}_{N A}(\vec{q})+\sum_{i<j=1}^{3}\left(\vec{j}_{g q \bar{q}}\left(\vec{r}_{i}, \vec{r}_{j}, \vec{q}\right)+\vec{j}_{\pi q \bar{q}}\left(\vec{r}_{i}, \vec{r}_{j}, \vec{q}\right)\right. \\
& \left.+\vec{j}_{\gamma \pi \pi}\left(\vec{r}_{i}, \vec{r}_{j}, \vec{q}\right)+\vec{j}_{\text {conf }}\left(\vec{r}_{i}, \vec{r}_{j}, \vec{q}\right)+\vec{j}_{\sigma}\left(\vec{r}_{i}, \vec{r}_{j}, \vec{q}\right)\right) .
\end{aligned}
$$

In our calculations the model wave function is the standard $S U(6) \otimes O(3)$, all the 
parameters are the same as in Ref. [1]], and the phase convention of Koniuk and Isgur [2, [3] is used. Our main purpose is to investigate the role of the two-body exchange currents, and spin-orbit and non-additive terms, in the photo-production of nucleon resonances. As a first step, we will neglect the configuration mixing effects. We also neglect the finite electromagnetic size of constituent quarks used in Ref. [9, 12]. In fact, this is easy to take into account, by multiplying every photo-production amplitude by a monopole form factor $F_{\gamma q}\left(\vec{q}^{2}\right)=1 /\left(1+(1 / 6) \vec{q}^{2} r_{\gamma q}^{2}\right)$.

All the calculations are made in the center mass frame and the results are shown in the Tables 目 and Tables [1]. The experimental data are given by the most recent Particle Data Review of Particle Physics [28]. We can see that the two-body exchange currents play an important role, especially the confinement current, in the photo-production of nucleon resonances. For the resonance $S_{11}(1535)$, in contrast to Ref. [3, [1], the theoretical overestimate versus experimental result does not exist anymore. It is interesting to notice that the Roper resonance $P_{11}(1440)$ agrees with experiment due to the two-body exchange currents, and not to the configuration mixing [4]. Another example is the $D_{15}(1675)$ proton excitation amplitudes $A_{1 / 2}$ and $A_{3 / 2}$, for which the two-body exchange currents give a nonzero contribution, although it is not in the right direction. However, for $P_{33}(1232)$, the theoretical result is still far below the experimental data because the two-body exchange currents cancel each other. Comparing with the large contributions to the $L=0$ and $L=1$ resonances, the contributions of the two-body exchange currents to the $L=2$ resonances are smaller, but they are still important to some $L=2$ resonances.

On the other side, we confirm the results of Close and $\mathrm{Li}$ [3], that the relativistic corrections $H_{\text {rel }}$ are important in most cases. Meyer et al. stressed the role of the twobody exchange currents on the photo-production of the resonance states with masses below $\sqrt{s}=1.6 \mathrm{GeV}$ [12], but the relativistic corrections $H_{\text {rel }}$ are neglected in their calculations. In fact, after considering the contributions of both the two-body exchange currents and $H_{\text {rel }}$, the agreement with experimental is improved, specially for the resonances $D_{13}(1520)$ and $P_{33}(1232)$. 


\section{THE NUCLEON SPIN STRUCTURE IN THE RESONANCE REGION}

The nucleon spin structure in the resonances region is mainly saturated by the nucleon resonances of photo- and electro-production. Extending the calculation of the photoproduction to electro-production is straightforward. However, the electro-production of resonances will become highly relativistic when the momentum transfer $q^{2}$ increases. We will follow the method of Li and Close [4] in order to remove the exponential decay with $q^{2}$. This method was first used by Foster and Hughes in order to study the electro-production in the equal-velocity frame [23].

In general, the nucleon spin structure function $g_{1}\left(x, Q^{2}\right)$ can be expressed as [24]

$$
g_{1}\left(\omega, Q^{2}\right)=\frac{M_{T} K}{8 \pi^{2} \alpha\left(1+\frac{Q^{2}}{\omega^{2}}\right)}\left[\sigma_{1 / 2}\left(\omega, Q^{2}\right)-\sigma_{3 / 2}\left(\omega, Q^{2}\right)+\frac{2 \sqrt{Q^{2}}}{\omega} \sigma_{T L}\left(\omega, Q^{2}\right)\right] .
$$

Here, $\sigma_{3 / 2}$ and $\sigma_{1 / 2}$ are the total cross sections, related to the excitation of hadronic states with helicity $3 / 2$ and $1 / 2$ respectively, $\sigma_{T L}$ is the longitudinal and transverse interference cross section, and $x=Q^{2} / 2 M_{T} \omega$ is the Bjorken scaling variable. Following Ref. [25], we choose the photon flux $K$ as "photon equivalent energy" $K=\left(W^{2}-M_{T}^{2}\right) / 2 M_{T}$, where $W$ is the total c.m. energy. Then we have for the first moment of the nucleon spin structure function

$$
\begin{aligned}
\Gamma\left(Q^{2}\right) & =\int_{0}^{x_{0}} d x g_{1}\left(x, Q^{2}\right) \\
& =\frac{Q^{2}}{16 \pi^{2} \alpha} \int_{\omega_{t h}}^{\infty} \frac{1-x}{\sqrt{\omega^{2}+Q^{2}}}\left[\sigma_{1 / 2}\left(\omega, Q^{2}\right)-\sigma_{3 / 2}\left(\omega, Q^{2}\right)+\frac{2 \sqrt{Q^{2}}}{\omega} \sigma_{T L}\left(\omega, Q^{2}\right)\right] \frac{d \omega}{\omega},
\end{aligned}
$$

where $\omega_{t h}$ is the threshold energy of pion photo-production, and $x_{0}=Q^{2} / 2 M_{T} \omega_{t h}$. In the large $Q^{2}$ limit, $\Gamma\left(Q^{2}\right) \rightarrow \Gamma$ (constant), and it is still positive at $Q^{2}=0.5 \mathrm{GeV}^{2}$ [18]. However, in the real photon limit, the cross section $\sigma_{T L}$ vanishes, and in fact the negative GDH sum rule [13] must be satisfied

$$
I\left(Q^{2}=0\right)=\int_{\omega_{t h}}^{\infty} \frac{d \omega}{\omega}\left[\sigma_{1 / 2}\left(\omega, Q^{2}=0\right)-\sigma_{3 / 2}\left(\omega, Q^{2}=0\right)\right]=-\frac{2 \pi^{2} \alpha}{M_{T}^{2}} \kappa^{2},
$$

where $\kappa$ is the anomalous magnetic moment of the nucleon, and $I\left(Q^{2}\right)=\left(2 M_{T}^{2} / Q^{2}\right) \Gamma\left(Q^{2}\right)$. Thus the nucleon spin structure is strongly $Q^{2}$ depended in the resonance region. 
With a Breit-Wigner parametrization in the resonance region, the absorption cross section can be expressed in terms of amplitudes as [26]

$$
\begin{aligned}
\sigma_{1 / 2}\left(\omega, Q^{2}\right) & =\sum_{R} \frac{2 M_{T}}{\left(W+W_{R}\right)} \frac{\Gamma_{R}}{\left(W-W_{R}\right)^{2}+\Gamma_{R}^{2} / 4}\left|A_{1 / 2}^{R}\left(\omega, Q^{2}\right)\right|^{2}, \\
\sigma_{3 / 2}\left(\omega, Q^{2}\right) & =\sum_{R} \frac{2 M_{T}}{\left(W+W_{R}\right)} \frac{\Gamma_{R}}{\left(W-W_{R}\right)^{2}+\Gamma_{R}^{2} / 4}\left|A_{3 / 2}^{R}\left(\omega, Q^{2}\right)\right|^{2}, \\
\sigma_{T L}\left(\omega, Q^{2}\right) & =\sum_{R} \frac{2 M_{T}}{\left(W+W_{R}\right)} \frac{\Gamma_{R}}{\left(W-W_{R}\right)^{2}+\Gamma_{R}^{2} / 4}\left[A_{1 / 2}^{R *} S_{1 / 2}^{R}+S_{1 / 2}^{R *} A_{1 / 2}^{R}\right]
\end{aligned}
$$

where, $\Gamma_{R}$ is the total decay width of the resonance $R$. The longitudinal transition amplitude is

$$
S_{1 / 2}=\left\langle R, J^{\prime}, \frac{1}{2}\left|H_{l}\right| N, \frac{1}{2}, \frac{1}{2}\right\rangle
$$

with the longitudinal transition operator

$$
H_{l}=\frac{1}{\sqrt{2 \omega}}\left(\epsilon_{0} j_{0}-\epsilon_{3} j_{3}\right)
$$

which is slightly different from Eq.(11) of Ref. [15] with a factor $1 / \sqrt{2 \omega}$, and which will lead to a more standard form of charge operators in the following. The longitudinal polarization vector can be chosen as

$$
\epsilon_{\mu}=\left(\epsilon_{0}, 0, \epsilon_{3}\right)=\left(\frac{q_{3}}{\sqrt{Q^{2}}}, 0,0, \frac{\omega}{\sqrt{Q^{2}}}\right) .
$$

Combining Eq.(19) with Eq. (20), we have

$$
S_{1 / 2}=\frac{\sqrt{Q^{2}}}{\omega \sqrt{2 \omega}}\left\langle R, J^{\prime}, \frac{1}{2}\left|j_{3}\right| N, \frac{1}{2}, \frac{1}{2}\right\rangle .
$$

Substituting Eq.(8) into the above equation, we obtain

$$
S_{1 / 2}=\frac{\sqrt{Q^{2}}}{|\vec{q}| \sqrt{2 \omega}} \sqrt{4 \pi} \sum_{J \geq 0} i^{J} \sqrt{2 J+1}\left\langle R, J^{\prime}, \frac{1}{2}\left|\hat{M}_{J m}(|\vec{q}|)\right| N, \frac{1}{2}, \frac{1}{2}\right\rangle .
$$

Also, according to the parity and angular momentum conservation, only one Coulomb multipole operator contributes to the longitudinal transition amplitude.

The charge operator, corresponding to the current in Eq.(4), is [27] 


$$
\begin{aligned}
\rho\left(\vec{r}_{i}, \vec{r}_{j}, \vec{q}\right) & =\sum_{i}\left[e_{i}+i \frac{e_{i}}{4 m^{2}} \vec{q} \cdot\left(\vec{\sigma}_{i} \times \vec{p}_{i}\right)\right] e^{i \vec{q} \cdot \vec{r}_{i}} \\
& -\sum_{i<j} \frac{i}{4 M_{T}}\left(\frac{\vec{\sigma}_{i}}{m}-\frac{\vec{\sigma}_{j}}{m}\right)\left[e_{i}\left(\vec{q} \times \vec{p}_{j}\right) e^{i \vec{q} \cdot \vec{r}_{i}}-e_{j}\left(\vec{q} \times \vec{p}_{i}\right) e^{i \vec{q} \cdot \vec{r}_{j}}\right],
\end{aligned}
$$

which includes both the spin-orbit and no-additive terms that are known to be important in reproducing the sum rule for the quantity $\sigma_{T L}$ [15]. The factor $1 / 2 \omega$, appeared in the charge operator of Ref. [15,27], is absorbed into Eq. (19), and the first term in the above equation is just the usual charge operator. All of those charge operators related to the two-body exchange currents are $O\left(1 / \mathrm{m}^{3}\right)$, and can be neglected in the calculation of $\sigma_{T L}$, although they are crucial in the case of the $\gamma N \rightarrow \Delta E 2$ transition [9] process and in reproducing the neutron charge radius [10].

In our calculations all the nucleon resonances with masses below $2.0 \mathrm{GeV}$ are included. Furthermore, in order to connect smoothly with the GDH sum rule $I\left(Q^{2}\right)$ at $Q^{2}=0$ and with the sum rules for $\Gamma_{p, n}\left(Q^{2}\right)$ at high $Q^{2}$, and based on the assumption that the main contribution comes from vector meson intermediate states at $Q^{2} \leq 2-3 \mathrm{GeV}^{2}$, the nonresonant part is modeled as in Ref. [14]:

$$
\begin{aligned}
I^{n r e s}\left(Q^{2}\right) & =2 m^{2} \Gamma\left(Q_{m}^{2}\right)\left[\frac{1}{Q^{2}+\mu^{2}}-\frac{c \mu^{2}}{\left(Q^{2}+\mu^{2}\right)^{2}}\right], \\
c & =1+\frac{\mu^{2}}{2 m^{2}} \frac{1}{\Gamma\left(Q_{m}^{2}\right)}\left[\frac{1}{4} \kappa^{2}+I^{\text {res }}(0)\right],
\end{aligned}
$$

where $\Gamma\left(Q_{m}^{2}\right)$ is a free parameter, $c$ is fixed by the value of the GDH sum rule at $Q^{2}=0$, and $\mu$ is the mass of $\rho$ meson. The extended GDH sum rule is simply expressed as a sum of the resonant part $I^{r e s}\left(Q^{2}\right)$ and the non-resonant part $I^{\text {nres }}\left(Q^{2}\right)$

$$
I\left(Q^{2}\right)=I^{r e s}\left(Q^{2}\right)+I^{\text {nres }}\left(Q^{2}\right)
$$

In fact, the importance of the non-resonant part was stressed early by Karliner [30], in order to reproduce the GDH sum rule at $Q^{2}=0 \mathrm{GeV}^{2}$. In our calculations, the chosen parameters are $\Gamma_{p}\left(Q_{m}^{2}\right)=0.237$ and $\Gamma_{n}\left(Q_{m}^{2}\right)=-0.025$, and $c_{p}=1.387$ and $c_{n}=-2.753$. These numbers were obtained using the experimental results for $\Gamma_{p, n}\left(Q^{2}\right)$ at $Q^{2}=2 \mathrm{GeV}^{2}$, and by the values of the GDH sum rules $I_{p, n}\left(Q^{2}=0\right)$, respectively. They are somewhat 
different from those of Ref. [14], but the changing in the shape of $\Gamma_{p, n}\left(Q^{2}\right)$ is weak in the low $Q^{2}$ region.

The $Q^{2}$ dependence of the first moment of the nucleon spin structure function $g_{1}\left(x, Q^{2}\right)$, both for the proton and the neutron in the resonance region, is shown in Figs. 1 and 2 . We include the resonant part calculation of Burkert and Ioffe [14], for comparison. The main difference between this work and ours is that we use the chiral constituent quark model, which in practice implies a different set of parameters, and we also include two-body exchange currents, although in contrast to the photo-production of nucleon resonances, in the case of the first moment of the nucleon spin structure function $g_{1}\left(x, Q^{2}\right)$ the effect of the two-body exchange currents is small.

For the proton or neutron separately, the contribution of the resonance $P_{33}(1232)$ is the dominant one. However, for the difference between the proton and the neutron, the contribution of this resonance is absent. In this case, chiral perturbation theory is useful, but it is valid only to as low as $0.2 \mathrm{GeV}^{2}$ [31]. In Fig. 3, we show our results for $\Gamma^{p}-\Gamma^{n}$, and we also compare them with the results of chiral perturbation theory [31]. Our results with only the resonant part have the correct slope and sign in the low $Q^{2}$ region.

In Figs. 7 and 5, we show our results for the $Q^{2}$ dependence of the GDH sum rule, both for the proton and the neutron, with the following extension:

$$
I_{G D H}\left(Q^{2}\right)=\frac{2 M_{T}^{2}}{Q^{2}} \int_{0}^{x_{0}} g_{1}\left(x, Q^{2}\right) d x .
$$

In our calculations, the sign-changing point of the generalized GDH sum rule for the proton appears around $Q^{2} \sim 0.27 \mathrm{GeV}^{2}$.

\section{SUMMARY}

Based on a chiral constituent quark model, we have calculated the photo-production amplitudes of all the nucleon resonances with masses below $2.0 \mathrm{GeV}$, and the first moment of the nucleon spin structure function $g_{1}\left(x, Q^{2}\right)$, including both relativistic corrections 
and those two-body exchange currents corresponding to the one-gluon exchange, one-pion exchange, one-sigma exchange and confinement potentials.

We find that the two-body exchange currents required by gauge invariance, specially the exchange current corresponding to the confinement potential, play an important role in most cases. But for $L=2$ nucleon resonances, the two-body exchange currents are less important. After taking into account the effects of both the two-body exchange currents and the relativistic corrections, the fit to experiment is improved.

It is known that the first moment of the nucleon spin structure function $g_{1}\left(x, Q^{2}\right)$ is strongly $Q^{2}$ depended below $Q^{2}=1.0 \mathrm{GeV}^{2}$, and we obtain a sign change at $Q^{2}=0.27 \mathrm{GeV}^{2}$. This strong $Q^{2}$ dependence means that in order to obtain a reliable theoretical prediction, the saturation of resonances should be performed carefully, and that is what we have done in this paper.

We mainly concentrated on the effects of the two-body exchange currents and relativistic effects, and did not consider configuration mixing effects. However, it is known that these effects have some contribution to resonance photo-production [4]. Furthermore, in order to compare with experiment, the $q^{2}$ dependence of helicity amplitudes also needs to be calculated. The confinement exchange current is also model dependent, and it is necessary to consider other possibilities, such as linear confinement and color screening potentials. All these effects will be included in future work.

\section{ACKNOWLEDGMENTS}

This research is supported by Fondecyt (Chile) postdoctoral fellowship 3000055 and project number 8000017, by a Cátedra Presidencial (Chile), and partly by the post Dr. foundation of SED of China. 


\section{REFERENCES}

[1] L. A. Copley, G. Karl, and E. Obryk, Nucl. Phys. B13, 303 (1969); R. P. Feynman, M. Kislinger, and F. Ravndal, Phys. Rev. D 3, 2706 (1971); F. E. Close, F. J. Gilman, and I. Karliner, D 6, 2533 (1972); F. J. Gilman and I. Karliner, Phys. Lett. B 46, 426 (1973); F. J. Gilman and I. Karliner, Phys. Rev. D 10, 2194 (1974).

[2] R. Koniuk and N. Isgur, Phys. Rev. D 18, 1888 (1980).

[3] F. E. Close and Z. P. Li, Phys. Rev. D 42, 2194 (1990).

[4] Z. P. Li and F. E. Close, Phys. Rev. D 42, 2207 (1990).

[5] A. De Rujula, H. Georgi and S.L. Glashow, Phys. Rev. D 12, 147 (1975).

[6] N. Isgur and G. Karl, Phys. Rev. D 18, 4187 (1978); D 19, 2653 (1979); D 20, 1191 (1979);

[7] For a review, see S.N. Mukherjee et al., Phys. Rep. 231, 201 (1993). For a recent discussion about quark orbit-spin splitting in meson and baryon systems, see e.g., P. R. Page, T. Goldman, and J. N. Ginocchio, Phys. Rev. Lett. 86, 204 (2001); N. Isgur, Phys. Rev. D 62, 054026 (2000); Phys. Rev. D 62, 014025 (2000).

[8] A. Manohar and H. Georgi, Nucl. Phys. B234, 189 (1984); L. Ya. Glozman and D. O. Riska, Phys. Rep. 268, 263 (1996).

[9] A. J. Buchmann, E. Hernández, and Amand Faessler, Phys. Rev. C 55, 448 (1997); A. J. Buchmann, U. Meyer, A. Faessler, and E. Hernandez, Phys. Rev. C 58, 2478 (1998).

[10] A. Buchmann, E. Hernández, and K. Yazaki, Phys. Lett. B 269, 35 (1991); Nucl. Phys. A569, 661 (1994).

[11] Georg Wagner, A. J. Buchmann, and Amand Faessler, Phys. Lett. B 359, 288 (1995); Phys. Rev. C 58, 3666 (1998); Phys. Rev. C 58, 1745 (1998); J. Phys. G 26, 267 (2000).

[12] U. Meyer, A. J. Buchmann, and A. Faessler, Phys. Lett. B 408, 19 (1997). 
[13] S. B. Gerasimov, Sov. J. Nucl. Phys. 2, 430 (1966); S. D. Drell and A. O. Hearn, Phys. Rev. Lett. 16, 908 (1966); For a recent review, see e.g., D. Drechsel, Prog. Part. Nucl. Phys. 34, 181 (1995); R. Pantforder, Ph. D. thesis, 1997, Bonn, hep-ph/9805434.

[14] V. Burkert and B. L. Ioffe, J. Exp. Theo. Phys. 78, 619 (1994); B. L. Ioffe, Yad. Fiz. 60, 1707 (1997); hep-ph/9709446.

[15] Z. P. Li and Y. B. Dong, Phys. Rev. D 54, 4301 (1996).

[16] W. X. Ma, D. H. Lu, A. W. Thomas, Z. P. Li, Nucl. Phys. A635, 497 (1998).

[17] D. Drechsel, S.S. Kamalov, G. Krein, and L. Tiator, Phys. Rev. D 59, 094021 (1999); D. Drechsel, S.S. Kamalov, and L. Tiator, Phys. Rev. D 63, 114010 (2001).

[18] K. Abe et al., Phys. Rev. Lett. 78, 815 (1997).

[19] V. Burkert and Zh. Li, Phys. Rev. D 47, 46 (1993); V. Burkert and B. L. Ioffe, Phys. Lett. B 296, 223 (1992).

[20] J. Soffer and O. Teryaev, Phys. Rev. Lett. 73, 3373 (1993); Phys. Rev. D 51, 25 (1995); Phys. Rev. D 56, 7458 (1997).

[21] S. J. Brodsky and J. Primack, Ann. Phys.(N.Y.) 69, 315 (1969).

[22] T. DeForest, Jr. and J. D. Walecka, Advances in Physics, 15, 1 (1966); T. W. Donnelly and A. S. Raskin, Ann. Phys.(N.Y.) 169, 247 (1986).

[23] F. Foster and G. Hughes, Z. Phys. C 14, 123 (1982).

[24] B. L. Ioffe, V. A. Khoze, and L. N. Lipatov, Hard processes(North-Holland, New York, 1983).

[25] D. Drechsel, O. Hanstain, S.S. Kamalov, and L. Tiator, Nucl. Phys. A645, 145 (1999).

[26] P. Stoler, Phys. Rep. 226, 103 (1993).

[27] F. E. Close and Z. P. Li, Phys. Lett. B 289, 143 (1992). 
[28] D. E. Groom et al., The European Physical Journal C 15, 1 (2000).

[29] K. Abe et al., Phys. Rev. D 58, 112003 (1998).

[30] I. Karliner, Phys. Rev. D 7, 2717 (1973).

[31] X. Ji, C. W. Kao, and J. Osborne, Phys. Lett. B 472, 1 (2000); V. Burkert, Phys. Rev. D 63, 097904 (2001). 


\section{TABLES}

TABLE I. Photo-production amplitudes of $\left[70,1^{-}\right]$resonance states in center mass frame in units of $\mathrm{GeV}^{-1 / 2} \times 10^{-3}$. $A_{T}$ shows the sum of all contributions. The experimental data are given by the most recent Particle Data Review [28].

\begin{tabular}{|c|c|c|c|c|c|c|c|c|c|c|c|}
\hline Multiple States & $A_{J}^{N}$ & $A_{N R}$ & $A_{S O}$ & $A_{N A}$ & $A_{g q \bar{q}}$ & $A_{\gamma \pi \pi}$ & $A_{\pi q \bar{q}}$ & $A_{\sigma}$ & $A_{\text {conf }}$ & $A_{T}$ & Exp. \\
\hline \multirow[t]{6}{*}[70,1^{-}]{$_{1} S_{11}(1535)$} & $A_{1 / 2}^{p}$ & 151 & -43 & 30 & -21 & 15 & -16 & 5 & -41 & 80 & $90 \pm 30$ \\
\hline & $A_{1 / 2}^{n}$ & -99 & 14 & 0 & 0 & -15 & 16 & -2 & 14 & -72 & $-46 \pm 27$ \\
\hline & $A_{3 / 2}^{p}$ & 94 & 27 & -19 & 22 & 14 & -20 & 0 & -1 & 117 & $166 \pm 5$ \\
\hline & $A_{3 / 2}^{n}$ & -93 & -9 & 0 & -22 & -14 & 20 & 0 & 0 & -118 & $-139 \pm 11$ \\
\hline & $A_{1 / 2}^{p}$ & -59 & 15 & -11 & -6 & 8 & -12 & -7 & 59 & -13 & $-24 \pm 9$ \\
\hline & $A_{1 / 2}^{n}$ & -16 & -5 & 0 & -3 & -8 & 11 & 2 & -20 & -39 & $-59 \pm 9$ \\
\hline \multirow[t]{2}{*}{$S_{11}(1650)$} & $A_{1 / 2}^{p}$ & 0 & 0 & 18 & 18 & 13 & -29 & 0 & 0 & 20 & $53 \pm 16$ \\
\hline & $A_{1 / 2}^{n}$ & 29 & -12 & -4 & -15 & -17 & 29 & 2 & -14 & -2 & $-15 \pm 21$ \\
\hline \multirow[t]{4}{*}{$D_{13}(1700)$} & $A_{3 / 2}^{p}$ & 0 & 0 & -33 & -36 & 13 & -15 & 0 & 0 & -71 & $-2 \pm 24$ \\
\hline & $A_{3 / 2}^{n}$ & -68 & 22 & 9 & 27 & -3 & 15 & -5 & 34 & 31 & $-3 \pm 44$ \\
\hline & $A_{1 / 2}^{p}$ & 0 & 0 & -19 & -17 & 0 & -12 & 0 & 0 & -48 & $-18 \pm 13$ \\
\hline & $A_{1 / 2}^{n}$ & -12 & 12 & 5 & 16 & 2 & 12 & -1 & 6 & 40 & $0 \pm 50$ \\
\hline \multirow[t]{4}{*}{$D_{15}(1675)$} & $A_{3 / 2}^{p}$ & 0 & 0 & 0 & -7 & 3 & -1 & 0 & 0 & -5 & $15 \pm 9$ \\
\hline & $A_{3 / 2}^{n}$ & -57 & 0 & 0 & 0 & 5 & 0 & -4 & 28 & -28 & $-58 \pm 13$ \\
\hline & $A_{1 / 2}^{p}$ & 0 & 0 & 0 & -5 & 2 & -1 & 0 & 0 & -4 & $19 \pm 8$ \\
\hline & $A_{1 / 2}^{n}$ & -41 & 0 & 0 & 0 & 4 & 0 & -3 & 20 & -20 & $-43 \pm 12$ \\
\hline$S_{31}(1650)$ & $A_{1 / 2}^{p, n}$ & 35 & 13 & 8 & 9 & 18 & -17 & -2 & 14 & 78 & $27 \pm 11$ \\
\hline \multirow[t]{2}{*}{$D_{33}(1700)$} & $A_{3 / 2}^{p, n}$ & 66 & -7 & -6 & 0 & 13 & -33 & 0 & 0 & 33 & $85 \pm 22$ \\
\hline & $A_{1 / 2}^{p, n}$ & 81 & -4 & -3 & -11 & 14 & -19 & 3 & -21 & 40 & $104 \pm 15$ \\
\hline \multirow[t]{2}{*}[70,0^{+}]{$_{2} P_{11}(1710)$} & $A_{1 / 2}^{p}$ & -25 & 28 & -2 & -6 & 0 & -1 & -4 & -24 & -34 & $9 \pm 22$ \\
\hline & $A_{1 / 2}^{n}$ & 8 & -9 & 0 & -1 & 0 & -1 & 1 & 8 & 6 & $-2 \pm 14$ \\
\hline
\end{tabular}


TABLE II. Photo-production amplitudes of $\left[56,2^{+}\right]$resonance states in center mass frame in units of $\mathrm{GeV}^{-1 / 2} \times 10^{-3}$. $A_{T}$ shows the sum of all contributions. The experimental data are given by the most recent Particle Data Review [28].

\begin{tabular}{|c|c|c|c|c|c|c|c|c|c|c|c|}
\hline Multiple States & $A_{J}^{N}$ & $A_{N R}$ & $A_{S O}$ & $A_{N A}$ & $A_{g q \bar{q}}$ & $A_{\pi q \bar{q}}$ & $A_{\gamma \pi \pi}$ & $A_{\sigma}$ & $A_{\text {conf }}$ & $A_{T}$ & Exp. \\
\hline \multirow[t]{8}{*}[56,2^{+}]{$_{2} P_{13}(1720)$} & $A_{3 / 2}^{p}$ & 32 & -26 & 2 & 5 & -8 & -1 & 0 & 5 & 9 & $-19 \pm 20$ \\
\hline & $A_{3 / 2}^{n}$ & 0 & 18 & 2 & -2 & 8 & 4 & 0 & -3 & -13 & $-29 \pm 61$ \\
\hline & $A_{1 / 2}^{p}$ & -116 & 46 & 4 & 12 & 0 & -6 & -2 & 34 & -28 & $18 \pm 30$ \\
\hline & $A_{1 / 2}^{n}$ & 41 & -31 & 4 & -4 & 0 & 8 & 2 & -23 & -3 & $1 \pm 15$ \\
\hline & $A_{3 / 2}^{p}$ & 64 & 35 & 3 & 9 & 4 & -5 & 0 & -6 & 104 & $133 \pm 12$ \\
\hline & $A_{3 / 2}^{n}$ & 0 & -23 & -3 & -3 & -4 & 5 & 0 & 4 & -24 & $-33 \pm 9$ \\
\hline & $A_{1 / 2}^{p}$ & -28 & 25 & 2 & -1 & 0 & 0 & -3 & 46 & 41 & $-15 \pm 6$ \\
\hline & $A_{1 / 2}^{n}$ & 49 & -16 & -2 & 0 & 0 & 0 & 2 & -31 & 2 & $29 \pm 10$ \\
\hline$P_{31}(1910)$ & $A_{1 / 2}^{p, n}$ & -26 & 0 & 0 & -3 & -6 & -4 & -1 & 16 & -24 & $3 \pm 14$ \\
\hline \multirow[t]{2}{*}{$P_{33}(1920)$} & $A_{3 / 2}^{p, n}$ & 45 & -20 & 2 & -3 & 4 & 5 & 2 & -25 & 10 & $23 \pm 17$ \\
\hline & $A_{1 / 2}^{p, n}$ & -26 & 34 & 3 & 0 & 5 & 0 & -1 & 10 & 25 & $40 \pm 14$ \\
\hline \multirow[t]{2}{*}{$F_{35}(1905)$} & $A_{3 / 2}^{p, n}$ & -71 & 49 & 4 & 1 & 3 & -3 & -3 & 36 & 16 & $-45 \pm 20$ \\
\hline & $A_{1 / 2}^{p, n}$ & -17 & 34 & 3 & 3 & 4 & -5 & -4 & 4 & 22 & $25 \pm 11$ \\
\hline \multirow[t]{2}{*}{$F_{37}(1950)$} & $A_{3 / 2}^{p, n}$ & -55 & 0 & 0 & -3 & -3 & 5 & -3 & 34 & -25 & $-97 \pm 10$ \\
\hline & $A_{1 / 2}^{p, n}$ & -42 & 0 & 0 & -3 & -2 & 4 & -3 & 26 & -20 & $-76 \pm 12$ \\
\hline \multirow[t]{2}{*}[56,0^{+}]{$_{2} P_{11}(1440)$} & $A_{1 / 2}^{p}$ & 38 & -34 & 7 & -20 & -15 & 5 & -19 & -27 & -65 & $-55 \pm 4$ \\
\hline & $A_{1 / 2}^{n}$ & -25 & 23 & -7 & 7 & 15 & -9 & 13 & 18 & 35 & $40 \pm 10$ \\
\hline \multirow[t]{2}{*}{$P_{33}(1600)$} & $A_{3 / 2}^{p, n}$ & -47 & 36 & -8 & -10 & 11 & -19 & -17 & -9 & -63 & $-9 \pm 21$ \\
\hline & $A_{1 / 2}^{p, n}$ & -27 & 21 & -5 & -6 & 6 & -11 & -10 & -5 & -37 & $-23 \pm 20$ \\
\hline \multirow[t]{2}{*}[56,0^{+}]{$_{0} P_{33}(1232)$} & $A_{3 / 2}^{p, n}$ & -180 & -12 & -12 & -19 & -33 & 27 & -21 & 68 & -182 & $-255 \pm 8$ \\
\hline & $A_{1 / 2}^{p, n}$ & -104 & -7 & -7 & -11 & -19 & 16 & -12 & 39 & -105 & $-135 \pm 5$ \\
\hline
\end{tabular}




\section{FIGURES}

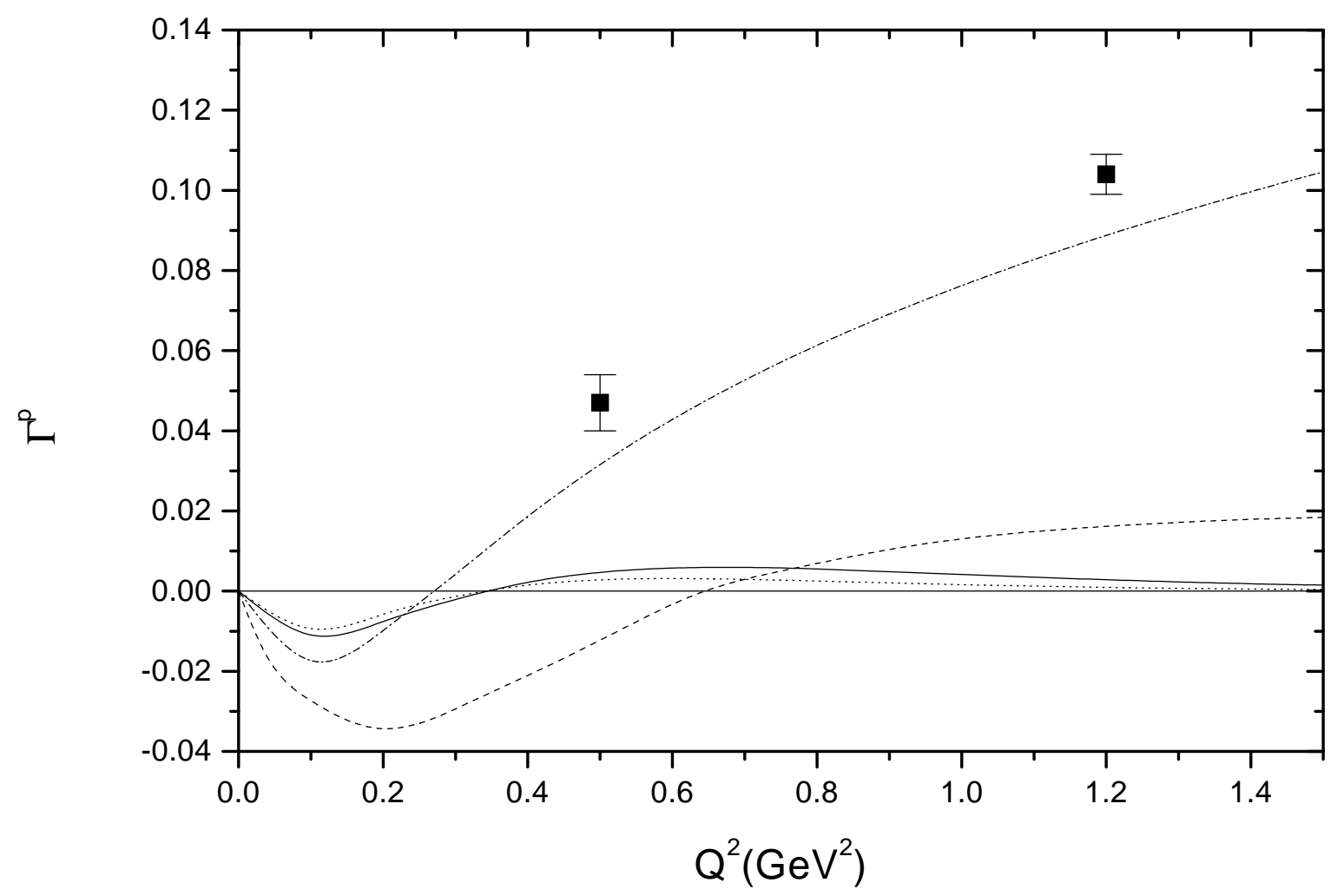

FIG. 1. The first moment of the spin structure function $g_{1}\left(x, Q^{2}\right)$ for proton. The solid and dotted lines show our calculation with and without the two-body exchange currents. The dashed line is the result of Ref. [14]. The dashed-dotted line is our result with the non-resonant part and the two-body exchange currents contributions. The experimental data are from [29]. 


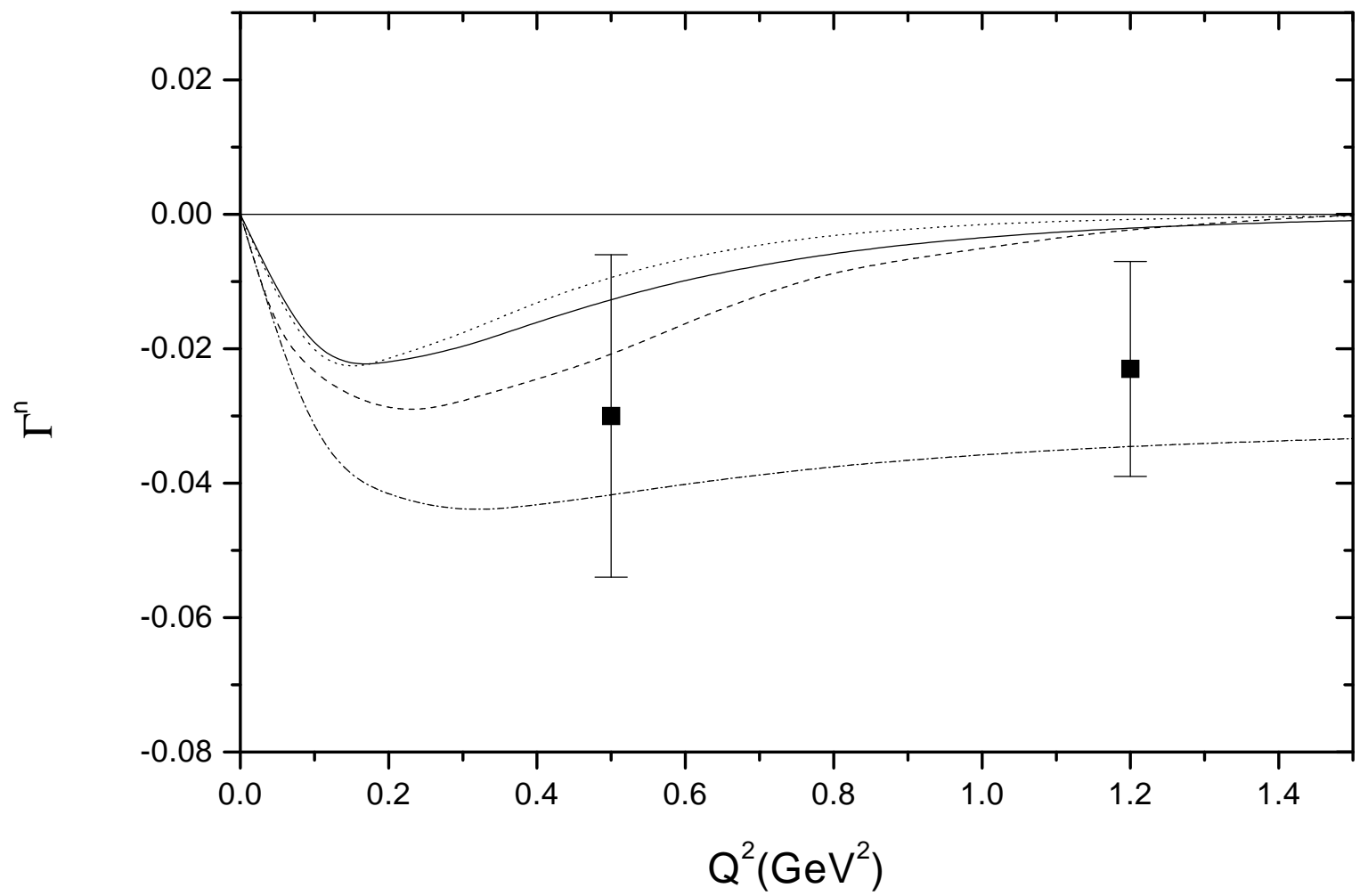

FIG. 2. The same as Fig. 1 for neutron. 


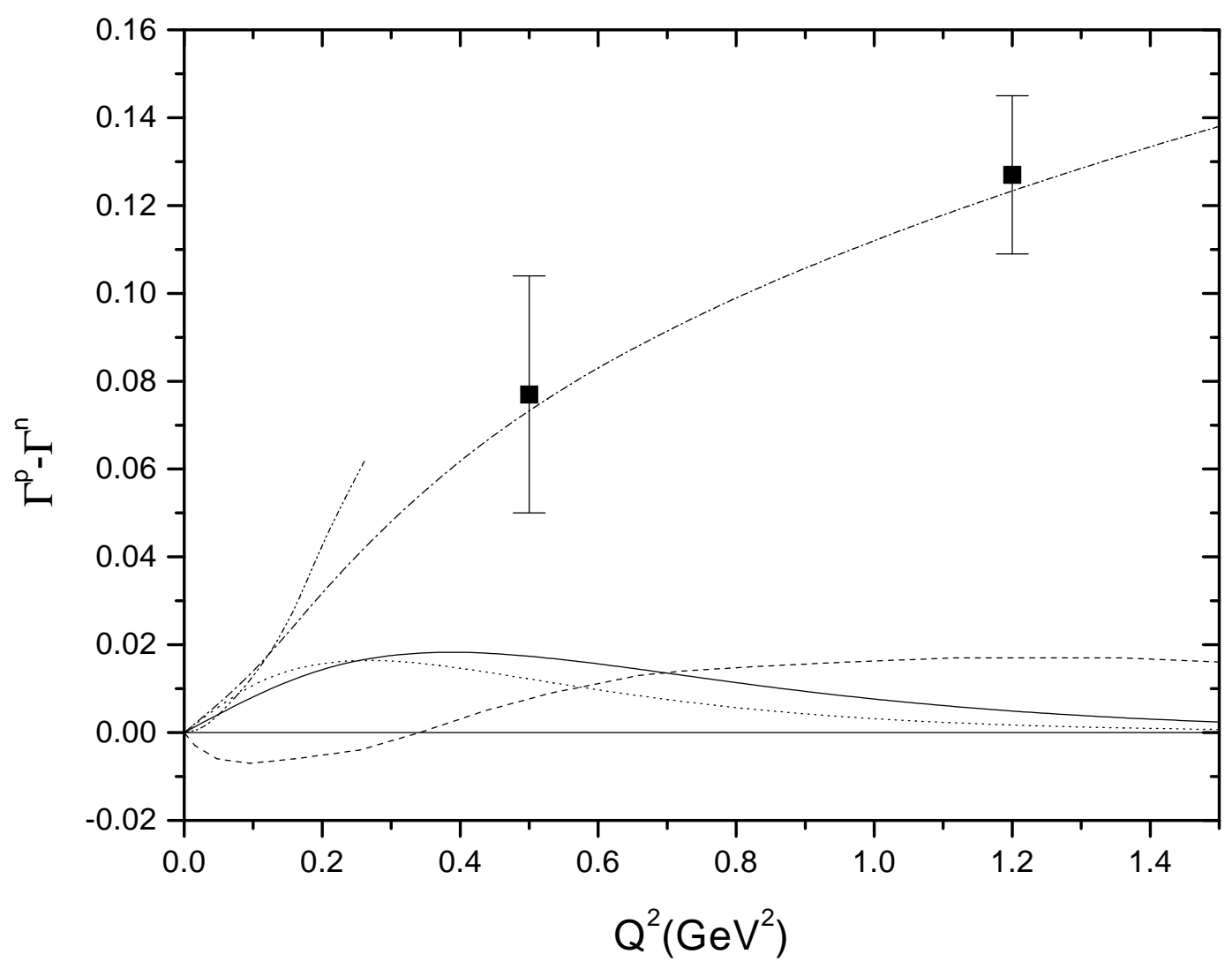

FIG. 3. The Bjorken integral $\Gamma_{p}-\Gamma_{n}$ of the spin structure function $g_{1}\left(x, Q^{2}\right)$. The solid and dotted lines show our calculation with and without two-body exchange currents. The dashed line is the result of Ref. [19]. The dashed-double dotted line is the $\chi \mathrm{PT}$ prediction [31]. Our result with the non-resonant part and the two-body exchange currents contributions is shown by the dashed-dotted line. The experimental data are from [29]. 


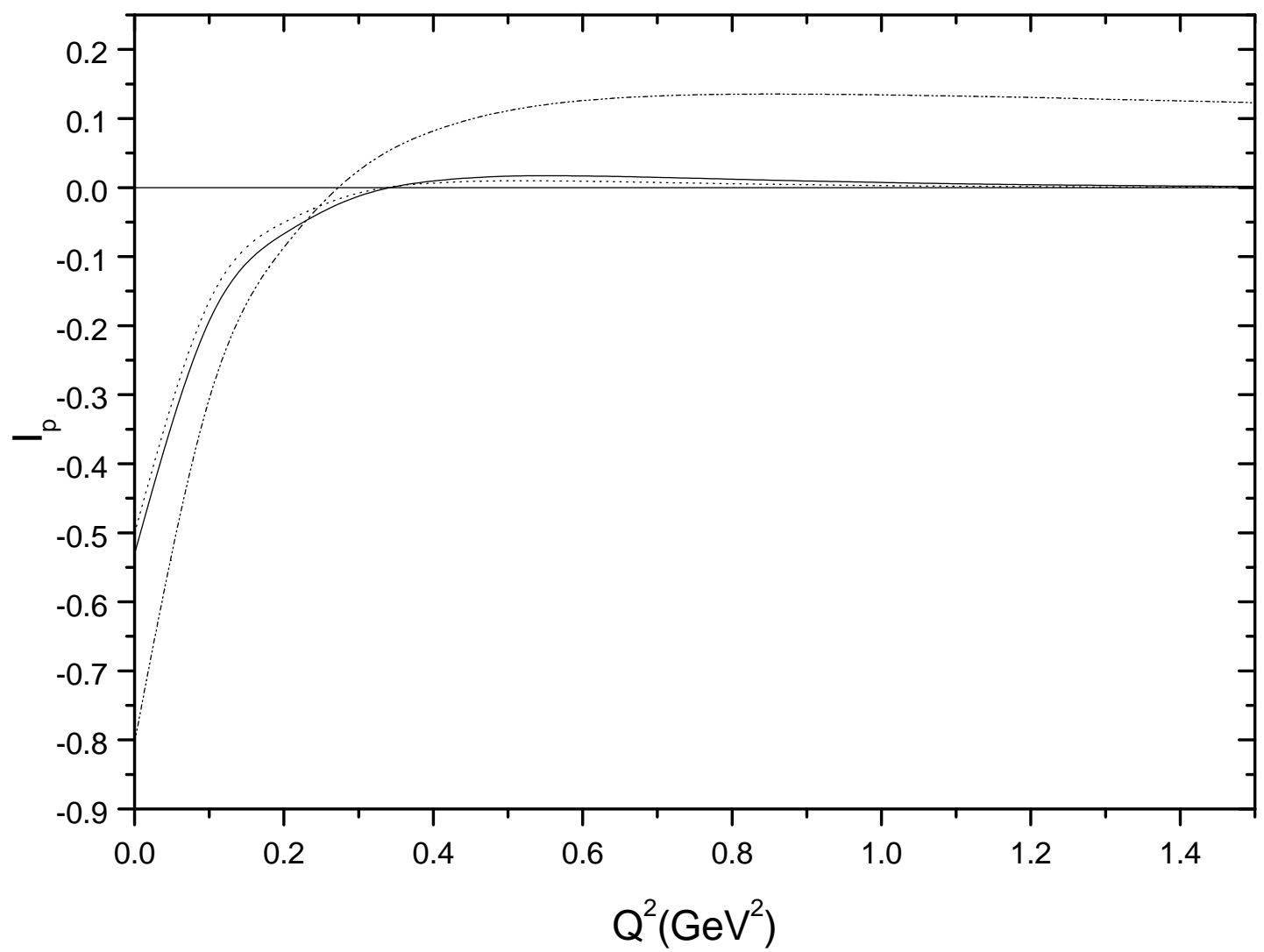

FIG. 4. The $Q^{2}$ dependence of GDH sum rule for proton. The solid and dotted lines show our calculation with and without the two-body exchange currents for proton. Our result with the non-resonant part and the two-body exchange currents contributions is shown by the dashed-dotted line. 


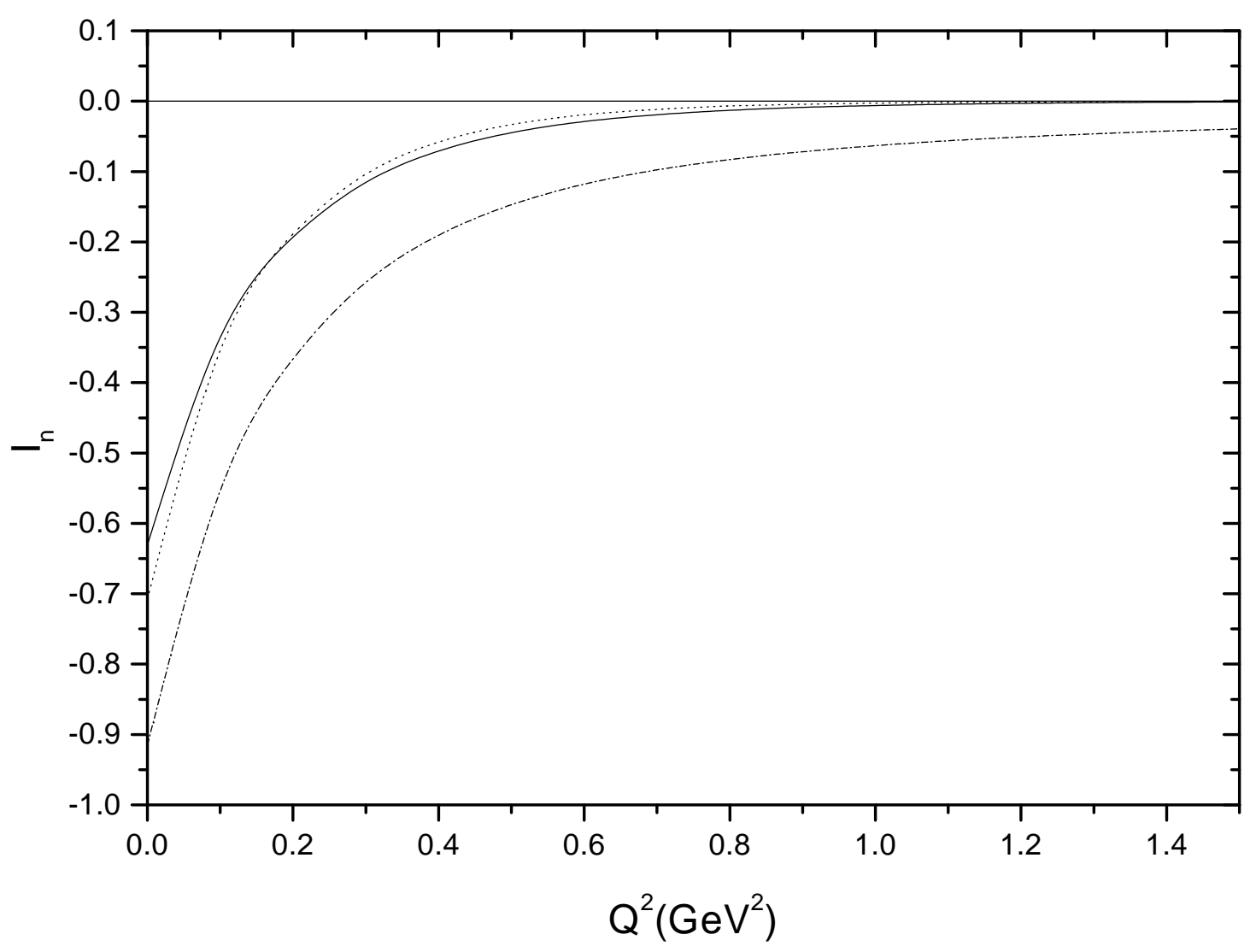

FIG. 5. The same as Fig. 1 for neutron. 